\title{
Lip Synchronization by Acoustic Inversion
}
Gregor Hofer*
University of Edinburgh

\author{
Korin Richmond ${ }^{\dagger}$
}
University of Edinburgh
Michael Berger
University of Edinburgh
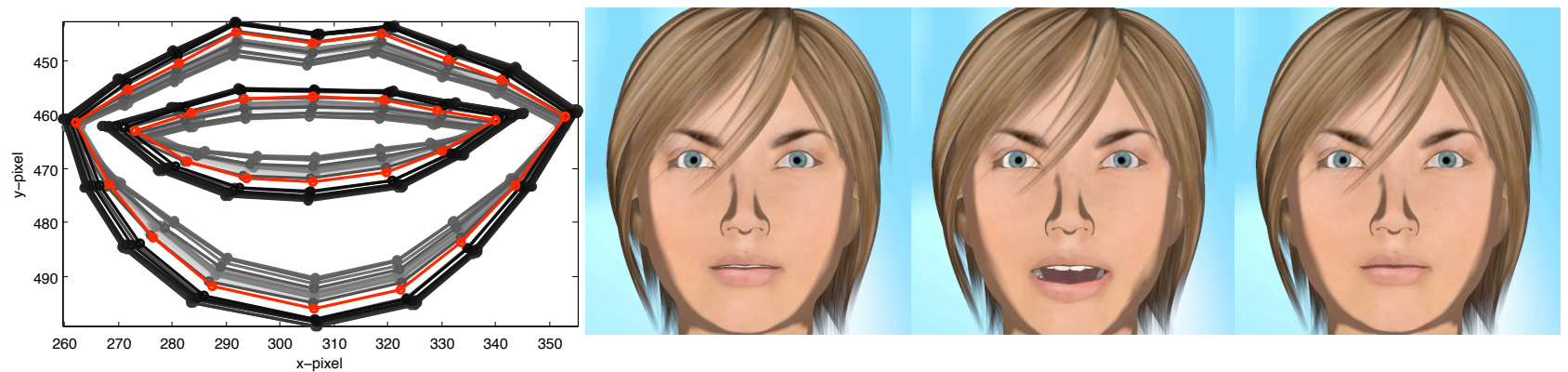

Figure 1: The word "how" as generated from our model (left) and the rendered equivalent sample frames (right). The red contours indicate the final frame, with frames going back in time represented with progressively lighter grayscale contours.

Keywords: lip synchronization, facial animation, neural networks

\section{Introduction}

Talking computer animated characters are a common sight in video games and movies. Although doing the mouth animation by hand gives the best results it is not always feasible because of cost or time constraints. Therefore producing lip animation automatically is highly desirable. The problem can therefore be phrased as mapping from speech to lip animation or in other words as an acoustic inversion. In our work we propose a solution that takes a sequence of input frames of speech and maps it directly to an output sequence of animation frames. The key point is that there is no need for phonemes or visemes which cuts one step in the usual lip synchronization process.

\section{Speech to motion mapping method}

At the heart of our mapping model is the mixture density network (MDN). The MDN can be considered as combining a trainable regression function (typically a non-linear regressor such as an artificial neural network) with a probability density function. In our work, we have been using a multilayer perceptron (MLP) as a trainable non-linear regressor and a Gaussian mixture model (GMM). An illustration is shown in Fig. 2. The role of the MLP is to take an input vector in one domain ( $\mathbf{x}$, acoustic features in this case) and map to the control parameters (priors, means and variances) of the pdf over the domain of the target parameters ( $t$, motion features). In this way, the MDN offers a model of probability density over the target domain conditioned on the input domain, $p(\mathbf{t} \mid \mathbf{x})$. Once trained, we input a sequence of acoustic feature vectors for an utterance and get as output a sequence of pdfs over the static motion features and their delta and deltadeltas. We then apply a maximum likelihood parameter generation algorithm (MLPG)[Tokuda et al. 2000] to this sequence of pdfs in order to obtain a single, most probable trajectory which optimizes the constraints between the distributions of static, delta and deltadelta features. This trajectory then drives the animation.

Trajectories can be generated in real time from the speech frames using a small amount of context frames. The model was trained

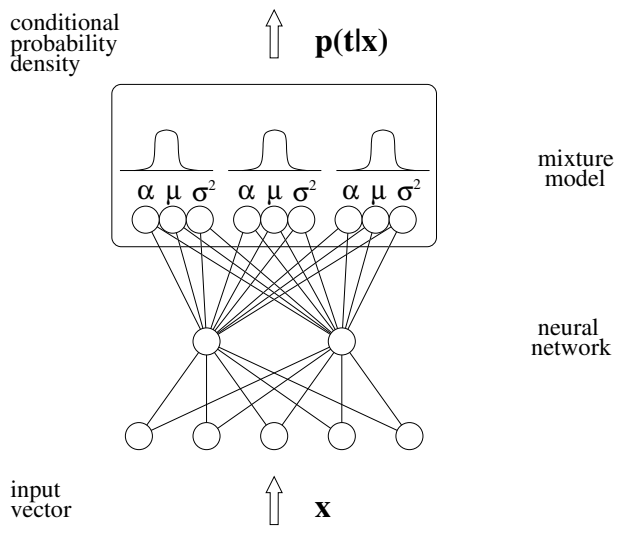

Figure 2: The mixture density network we use combines a multilayer perceptron and Gaussian mixture model. We input speech frames and get distributions over animation frames.

on the first 4 PCA components of 28 tracked points around the mouth. The total amount of training data was 207 utterances $(\sim 25$ min.). The input speech features were the first 25 mel cepstrum coefficients, which are standard speech recognition features. A short perceptual evaluation was carried out with 17 subjects judging 5 utterances. No significant difference between the preference for animation from the original data $(46 \%)$ and animation from the model (54\%) was found, which is a promising result to further develop MDNs for lip synchronization.

\section{References}

Tokuda, K., Yoshimura, T., Masuko, T., Kobayashi, T., AND KitAMURA, T. 2000. Speech parameter generation algorithms for HMM-based speech synthesis. In Proc. ICASSP, 1315-1318.

\footnotetext{
*e-mail: ghofer@inf.ed.ac.uk

†e-mail:korin@cstr.ed.ac.uk

‡e-mail:m.a.berger@sms.ed.ac.uk
} 